\title{
Spectral Efficiency and Bit Error Rate Analysis of WiMAX Using Diverse Modulation Techniques over Rayleigh Channel
}

\author{
Bindu Handa*, Anita Suman**, Praveen Kumar*** \\ *(Department of Electronics and communication, BCET, Punjab, India handa.bindu@gmail.com) \\ ** (Department of Electronics and communication, BCET, Punjab, India suman_al @yahoo.com) \\ *** (Department of Electronics and communication, BCET, Punjab, India parveen.klair@gmail.com) \\ Corresponding author: Bindu Handa
}

\begin{abstract}
Worldwide Interoperability for Microwave Access (WiMAX) is a popular wireless technology which offers fast voice, video and information benefits up to the client end. In this paper, the OFDM based WiMAX physical layer makes use of concatenated Reed Solomon Convolutional (RS-CC) code and different modulation techniques with different code rates along with different cyclic prefix. As WiMAX can be used for NLOS (Non Line of Sight) communication so Rayleigh channel has been used as transmission medium. The comparison of various cyclic prefix such as 1/4, 1/8, 1/16 and 1/32 has been done on different modulation techniques over Rayleigh channel. The performance of OFDM based WiMAX physical layer has been analyzed from the graphs between BER (Bit Error rate) vs. SNR (Signal to Noise Ratio) and Spectral Efficiency vs. SNR (Signal to Noise Ratio) for different modulation techniques. The simulation results of BER vs. SNR conclude that different modulations and code rate provide better performance at guard time interval of $1 / 32$. The simulation result of Spectral efficiency vs SNR conclude that 64 QAM with code rate $3 / 4$ has highest spectral efficiency because of which it can be used for transmitting larger amount of data with lesser amount of transmission error.
\end{abstract}

Keywords: AWGN, BER, Rayleigh, Spectral efficiency, WiMAX

Date of Submission: $11-07-2017$

Date of acceptance: 20-07-2017

\section{INTRODUCTION}

WiMAX is shortened form of Worldwide Interoperability for Microwave Access. It is also known by name IEEE 802.16. WiMAX depends on wireless metropolitan area networking (WMAN) standards created by the IEEE 802.16 group embraced by both IEEE and the ETSI HIPERMAN group [1]. The IEEE 802.16 group was shaped in 1998 to build up an air-interface standard for remote broadband. The physical layer of WiMAX relies on orthogonal frequency division multiplexing which allow WiMAX to works in NLOS state. It supports high peak data rate with scalable bandwidth, TDD (Time Division duplexing), FDD (Frequency Division duplexing) and advanced antenna techniques. The WiMAX takes full favorable position of link adaption technique along with coding.

\section{BLOCK DIAGRAM OF WIMAX}

The Block diagram of OFDM based WiMAX physical layer comprises of different blocks that plays an important role. The modulation and OFDM multiplexer are the important building blocks of the WiMAX Physical layer. The block diagram of WiMAX physical layer is shown in Fig.1.

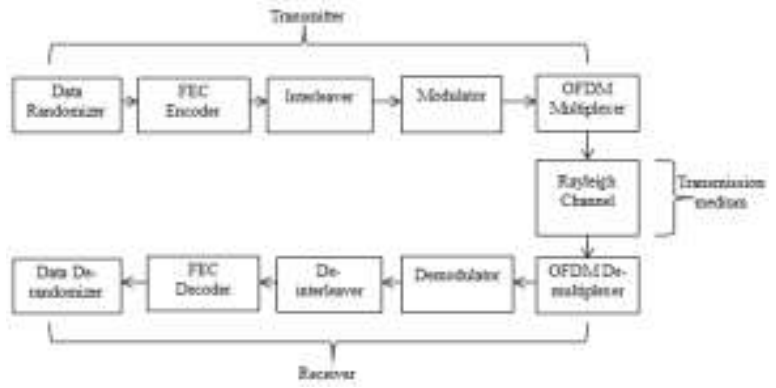

Fig. 1 Block diagram of physical layer of WiMAX

\subsection{Randomizer and De-Randomizer}

Randomization provides security by removing long arrangements of successive ones or sequential zeros. Data randomization is carried out on both uplink and downlink burst of data [1]. If the measure of data that is to be transmitted does not fit precisely with the measure of data apportioned, then cushioning of $0 \_F F$ is added to the end of the transmission block. For data randomization in WiMAX, Pseudo Random binary Sequence generator is used with 15-bits feedback polynomial $\mathrm{X}^{15}+\mathrm{X}^{14}+1$ which produces pseudo-noise sequence of length $32767\left(2^{15}-1\right)$. The Pseudo Random binary Sequence generator is shown in Fig. 2. 


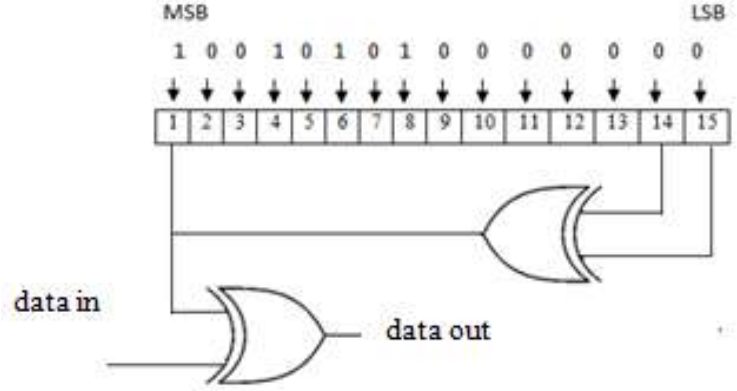

Fig. 2 Data randomizer

\subsection{FEC Encoder and FEC Decoder}

FEC stands for forward error correction. FEC encoder is used for adding redundancy in a controlled way which will enhance the reliability of data [1] at the transmitter. In OFDM based WiMAX physical layer, Concatenated Reed-Solomon Convolutional Code (RS-CC) is used and is required on both the downlink and uplink. Reed Solomon code act as outer code and Convolutional code act as inner code as shown in Fig.3.

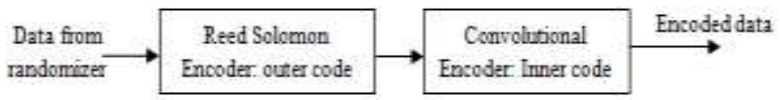

Fig. 3 Concatenated RS-CC Encoder

The encoding process is performed by first passing the data in the form of block through the RS (Reed Solomon) encoder and then passing it through a CC (convolutional) encoder. A single 0x00 tail byte is annexed to the end of each burst. After randomization, this tail byte is done. In the Reed Solomon encoder, the repetition bits are transmitted before the input bits, keeping the 0x00 tail byte toward the end of the allotment. When the aggregate number of data bits in a burst is not an integer number of bytes, zero pad bits are included after the zero tail bits. These zero pad bits are not randomized. The Reed Solomon-Convolutional Code encoding process is illustrated in Fig.4. Keeping in mind the end goal to accomplish code rates higher than $1 / 2$, the yield of the encoder is punctured utilizing puncturing pattern as shown in Table 1. FEC decoder is used to diminish the redundancy that is included at the transmitter. This redundancy is investigated to see if there are any errors.

\subsection{Interleaver and De-interleaver}

Interleaver is used to permute the encoded data which is the output of FEC encoder. It is utilized to conquer very long successions of errors. The span of interleaving block relies on the number of coded bits per encoded block size. The interleaving process is done utilizing a two-stage permutation process.

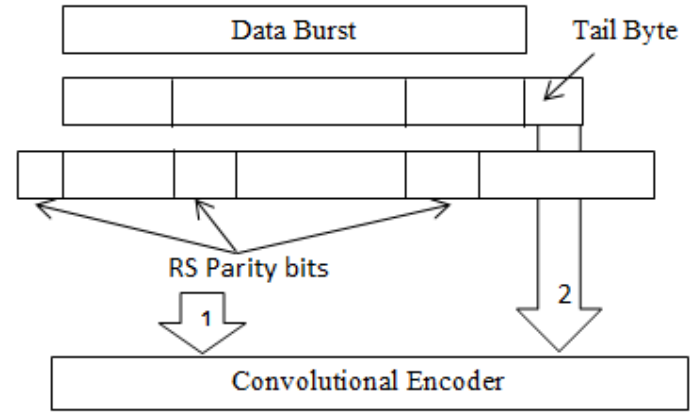

Fig. 4 RS-CC encoding process

Table 1 . Puncturing configuration of CC code

\begin{tabular}{|c|c|c|c|c|}
\hline Rate & $d_{\text {free }}$ & $\mathrm{X}_{\mathrm{O} / \mathrm{P}}$ & $\mathrm{Y}_{\mathrm{O} / \mathrm{P}}$ & $\begin{array}{c}\mathrm{XY} \text { (Punctured } \\
\mathrm{O} / \mathrm{P})\end{array}$ \\
\hline $1 / 2$ & 10 & 1 & 1 & $\mathrm{X}_{1} \mathrm{Y}_{1}$ \\
\hline $2 / 3$ & 6 & 10 & 11 & $\mathrm{X}_{1} \mathrm{Y}_{1} \mathrm{Y}_{2}$ \\
\hline $3 / 4$ & 5 & 101 & 110 & $\mathrm{X}_{1} \mathrm{Y}_{1} \mathrm{Y}_{2} \mathrm{X}_{3}$ \\
\hline $5 / 6$ & 4 & 10101 & 11010 & $\mathrm{X}_{1} \mathrm{Y}_{1} \mathrm{Y}_{2} \mathrm{X}_{3} \mathrm{Y}_{4} \mathrm{X}_{5}$ \\
\hline
\end{tabular}

In first permutation, the adjacent coded bits are mapped onto nonadjacent subcarriers and are characterized by the following equation 1 :

$$
\mathrm{m}_{\mathrm{k}}=\left(\mathrm{N}_{\mathrm{cbps}} / 12\right) \times \bmod (\mathrm{K}, 12)+\text { floor }(\mathrm{K} / 12)
$$

where ' $m_{k}$ ' is the Index of coded bits after first permutation, ' $\mathrm{N}_{\mathrm{cbps}}$ ' is the number of coded bits per symbol and ' $\mathrm{K}$ ' is the Index of coded bits before first permutation.

The adjacent coded bits are mapped alternately onto less or more significant bits of the constellation in second permutation. It is characterized by the equation 2 and equation 3 :

$\mathrm{s}=\operatorname{ceil}\left(\mathrm{N}_{\mathrm{cpc}} / 2\right)$

$\mathrm{j}_{\mathrm{k}}=\mathrm{s} \times$ floor $\left(\mathrm{m}_{\mathrm{k}} / \mathrm{s}\right)+\left(\mathrm{m}_{\mathrm{k}}+\mathrm{N}_{\mathrm{cbps}}-\right.$ floor $\left(12 \times \mathrm{m}_{\mathrm{k}} /\right.$

$\left.\left.\mathrm{N}_{\text {cbps }}\right)\right) \bmod (\mathrm{s})$

where 'Ncpc' is the number of coded bits per carrier, ' $\mathrm{j}_{\mathrm{k}}$ ' is the Index of coded bits after second permutation.

De-interleaver is utilized to undo the changes done by interleaver at the transmitter side to retrieve the actual information.

\subsection{Modulator and Demodulator}

After interleaving, the next block of WiMAX physical layer is modulator. WiMAX utilizes adaptive modulation where modulation changes relying on the conditions of channel. Demodulation block at the receiver side is used to demodulate the signal waveform back to digital data. 

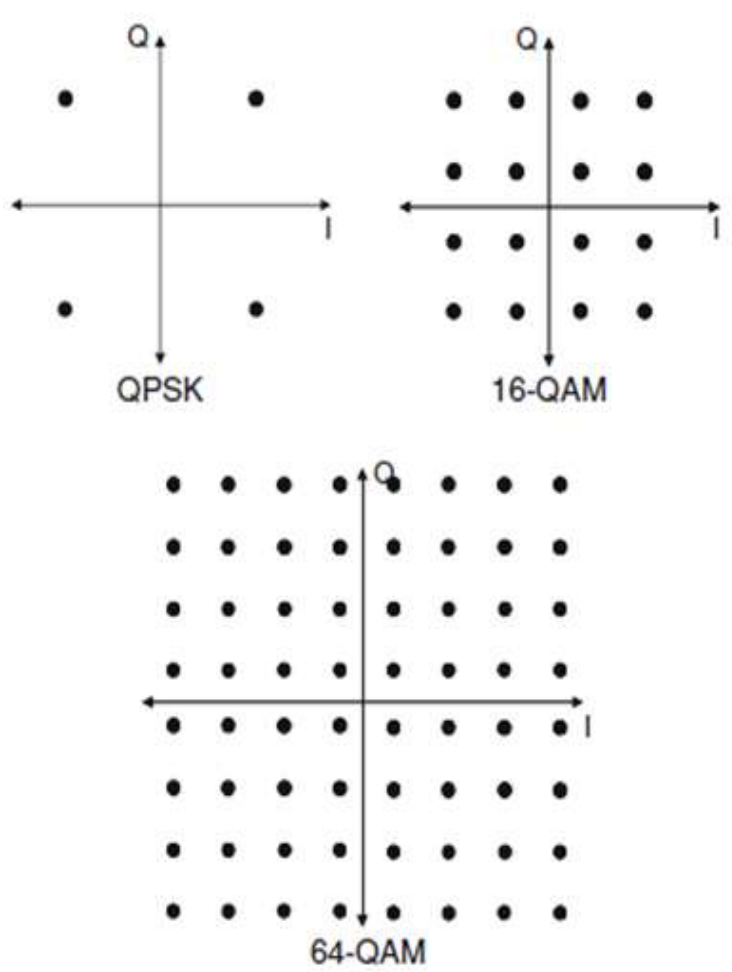

Fig. 5 QPSK, 16 QAM and 64 QAM constellations

\subsection{OFDM multiplexer and De-multiplexer}

OFDM is short for orthogonal frequency division multiplexing. Here, the modulated data is converted into time domain from frequency domain. This is done by employing IFFT (Inverse Fast Fourier Transform or IDFT (Inverse Discrete Fourier Transform). Also cyclic prefix has been included with the time domain data to diminish inter-symbol interference (ISI). There are four different duration of cyclic prefix that are available in the standard. The different cyclic prefix are 1/4, 1/8, 1/6 and 1/32. Then it is transmitted through the channel. OFDM de-multiplexer evacuates cyclic prefix which is included at the transmitter for reducing Inter-symbol interference. The received signal is changed into frequency domain utilizing FFT (Fast Fourier Transform) or DFT (Discrete Fourier Transform) algorithm. As OFDM symbol comprises of data, pilots and a zero DC subcarrier with guard bands. Pilot carriers and data values are extricated at the receiver side.

\subsection{Channel}

A channel or physical medium is the transmission way over which a signal proliferates. Numerous transmission media are utilized as communications channel. Transmission media are categorized as guided media and unguided media. The channel used in this paper is Rayleigh fading channel. Rayleigh channel is a multipath fading channel where there is huge number of reflection present. Mathematically, Rayleigh fading channel is represented as:

$\mathrm{r}(\mathrm{t})=\mathrm{s}(\mathrm{t}) \times \mathrm{h}(\mathrm{t})+\mathrm{n}(\mathrm{t})$

where $r(t)$ is the received signal, $s(t)$ is the transmitted signal, $\mathrm{h}(\mathrm{t})$ is the random channel matrix and $n(t)$ is the background noise as shown in Fig. 6 .

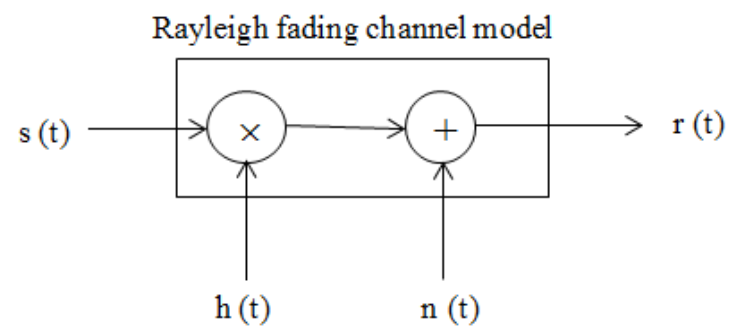

Fig. 6 Rayleigh channel model

\section{RELATED WORK}

Mondal et al. (2009) [3] evaluates the performance of a WiMAX system under different digital modulation (BPSK, QPSK, 4-QAM and 16QAM) and different communication channels (AWGN, Rayleigh and Rician channel) [3]. The simulation results of bit error rate (BER) states that the implementation of interleaved RS code $(255,239,8)$ with $2 / 3$ rated convolutional code under BPSK modulation technique is exceedingly compelling to battle in the WiMAX communication system. Md. Anamul Islam et al. (2010) [4] uses concatenated cyclic redundancy check and convolution code (CRC-CC) along with AWGN and frequency flat fading channels. The system is analyzed on the basis of BER and spectral efficiency [4]. The results of BER and spectral efficiency illustrate that the system performance is exceedingly reliant on channel conditions and can be streamlined by AMC technique. Under AWGN and Rician channel, 64 QAM with 3/4 rated coding show lower BER whereas, in Rayleigh channel, 16-QAM shows lower BER. 16 QAM with 2/3 rated code battles exceptionally compelling spectrum efficiency under all mobile environments. M.A.Mohamed et al. (2010) [5] analyzed the WiMAX physical layer under different combinations of digital modulation schemes and different communication channels. The performance of the simulated system was evaluated using the bit-error-rate (BER) [5]. This paper stated that when channel conditions are poor, energy efficient schemes such as BPSK or QPSK were used and when channel quality improves, higher M-ary modulation schemes such as16-QAM or 64-QAM was used. Prabhakar Telagarapu et al. (2011)[6] analyzed the performance of WiMAX physical layer by using Reed Solomon and Convolutional code as FEC (Forward Error Correction) code with cyclic 
prefix and interleaving using different modulation techniques [6]. The performance of WiMAX physical layer is evaluated on the basis of BER vs. SNR. The results are shown in the paper as with and without FEC for different modulation techniques. A. Islam et al. (2012) [7] compared the performance of concatenated CRC-CC (Cyclic Redundancy check and convolutional) and RS-CC (Reed Solomon and Convolutional) codes over AWGN and multipath fading channels. The simulation results concluded that the performance of concatenated $\mathrm{CRC}-\mathrm{CC}$ is better when contrasted with concatenated RS-CC code under QAM over AWGN and multipath fading channels. Abdul Rehman et al. (2012) [8] used Reed Solomon encoder with Convolutional encoder as FEC code. AWGN and fading channels are used along with adaptive modulation technique to evaluate the performance of WiMAX system. The Simulation result concluded that the implementation of interleaved RS code with $2 / 3$ rated convolutional code under BPSK modulation technique is more effective in the WiMAX system. M. M. Nuzhat Tasneem Awonet al. (2012) [9] evaluated the BER performance of WiMAX system on the basis of different encoding rates and different modulation techniques over AWGN, Rayleigh and Rician channel. The results show that AWGN channel performance is best among all the channels whereas the performance of Rayleigh channel is the worst of all the channels. The performance of Rician channel is worse than that of AWGN channel but better than Rayleigh channel. Karrar AlSlmman et al.(2012) [10] compared LDPC (Low Density Parity Check) code with commonly used concatenated RS-CC (Reed Solomon-Convolutional) code over standard AWGN channel. The simulation result shows that the performance of LDPC code is better than RS-CC code. Kushwah et al.(2013) [11] demonstrated WiMAX physical layer model by using MATLAB simulink. This paper is used to illustrate the effect of different modulation techniques, coding rates, cyclic prefix and OFDM symbols on the performance of WiMAX physical layer system. Manju Agrawal et al.( 2015)[12] evaluated the WiMAX system performance with the help of BPSK, QPSK, 8QAM, 16-QAM, 64-QAM and 256-QAM modulation techniques on the basis of BER, Signal to Noise Ratio and Spectral Efficiency. The Simulation results stated that 256-QAM performs better with highest SNR and BPSK provide better performance with lowest SNR. Also 256-QAM can be used for transmitting maximum amount of data with minimum amount of error.

\section{SIMULATION RESULTS}

The simulation results consists of Bit Error rate (BER) vs. Signal to Noise Ratio (SNR) graphs and Spectral efficiency vs. Signal to Noise Ratio (SNR) graph of OFDM based WiMAX physical layer over Rayleigh channel using diverse modulation techniques. Some light will be tossed on each of this one by one. The performance of OFDM based WiMAX physical layer system over Rayleigh channel using diverse modulation techniques and cyclic prefix $1 / 4$ is depicted in Fig. 7. After analyzing this figure, we get that BER of $10^{-3}$ is achieved in case of QPSK $1 / 2$ at SNR of $25.24 \mathrm{~dB}$ whereas in case of QPSK $3 / 4$, the same is obtained at SNR of $25.42 \mathrm{~dB}$. Similarly in case of 16 QAM $3 / 4$, 64 QAM $2 / 3$ and 64 QAM 3/4, the BER of $10^{-3}$ is obtained at SNR of $20.73 \mathrm{~dB}, 20 \mathrm{~dB}$ and $21.13 \mathrm{~dB}$ respectively. From Fig.8, we get that BER of $10^{-3}$ is achieved in case of QPSK $1 / 2$ at SNR of $24.15 \mathrm{~dB}$ whereas in case of QPSK 3/4, the same is obtained at SNR of $24.62 \mathrm{~dB}$. Similarly in case of 16 QAM $3 / 4$, 64 QAM 2/3 and 64 QAM 3/4, the BER of $10^{-3}$ is obtained at SNR of $19.70 \mathrm{~dB}, 19.54 \mathrm{~dB}$ and 20.24 dB respectively. Fig.9 depicts the BER of WiMAX using different modulation techniques with cyclic prefix 1/16 over Rayleigh channel. After analyzing this figure, we get that BER of $10^{-3}$ is achieved in case of QPSK $1 / 2$ at SNR of $23.10 \mathrm{~dB}$ whereas in case of QPSK $3 / 4$, the same is obtained at SNR of $23.92 \mathrm{~dB}$. Similarly in case of 16 QAM $3 / 4,64$ QAM $2 / 3$ and 64 QAM $3 / 4$, the BER of $10^{-3}$ is obtained at SNR of $18.92 \mathrm{~dB}, 18.63 \mathrm{~dB}$ and $19.30 \mathrm{~dB}$ respectively. After analyzing Fig. 10, we get that BER of $10^{-3}$ is achieved in case of QPSK $1 / 2$ at SNR of $22.22 \mathrm{~dB}$ whereas in case of QPSK $3 / 4$, the same is obtained at SNR of $23.01 \mathrm{~dB}$. Similarly in case of 16 QAM 3/4, 64 QAM 2/3 and 64 QAM 3/4, the BER of $10^{-3}$ is obtained at SNR of $18.10 \mathrm{~dB}, 18.04 \mathrm{~dB}$ and $18.30 \mathrm{~dB}$ respectively. The performance of different modulations i.e. QPSK 1/2, QPSK 3/4, 16 QAM 3/4, 64 QAM 2/3, and 64 QAM $3 / 4$ using cyclic prefix $1 / 4$, $1 / 8,1 / 16$ and $1 / 32$ is depicted in Fig. 11, Fig.12, Fig.13, Fig.14 and Fig.15 respectively. The Table that outlines all the BER results of different modulation along with different cyclic prefix is given in Table 2.The Table that outlines the spectral Efficiency of WiMAX over Rayleigh channel is given in Table 3. 


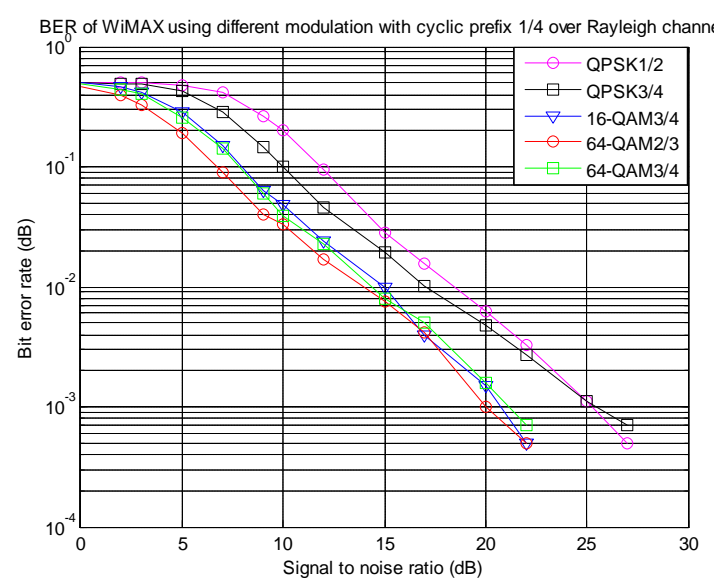

Fig. 7 BER of WiMAX with cyclic prefix 1/4

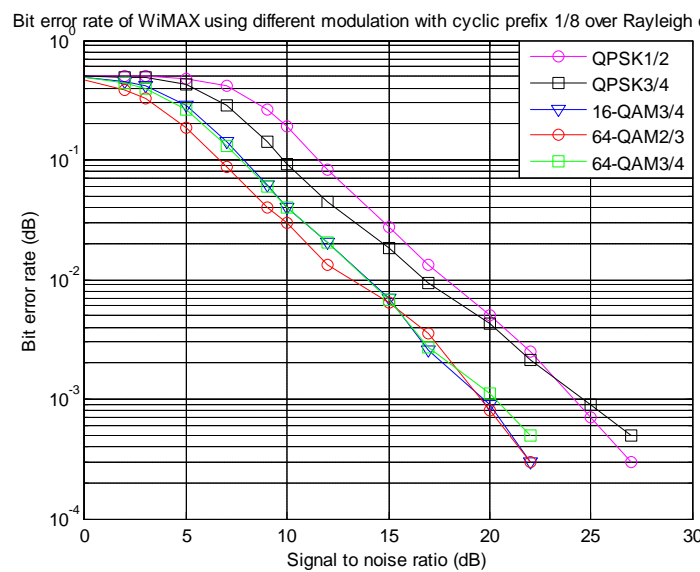

Fig. 8 BER of WiMAX with cyclic prefix 1/8

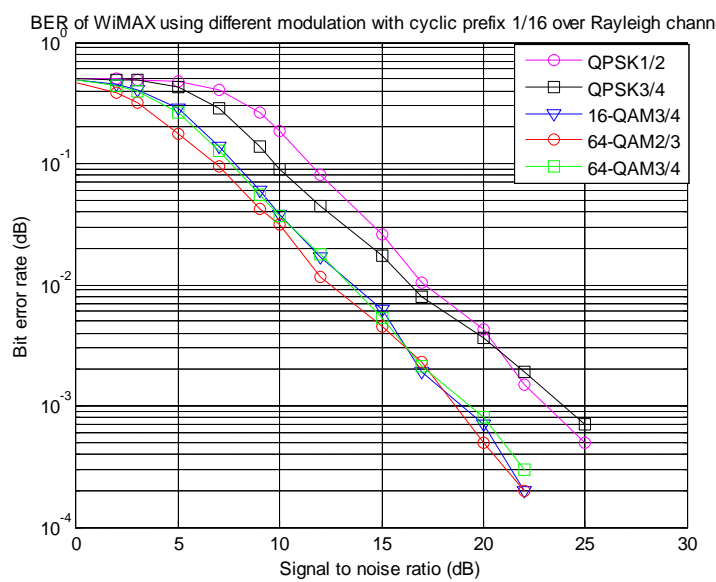

Fig. 9 BER of WiMAX with cyclic prefix 1/16

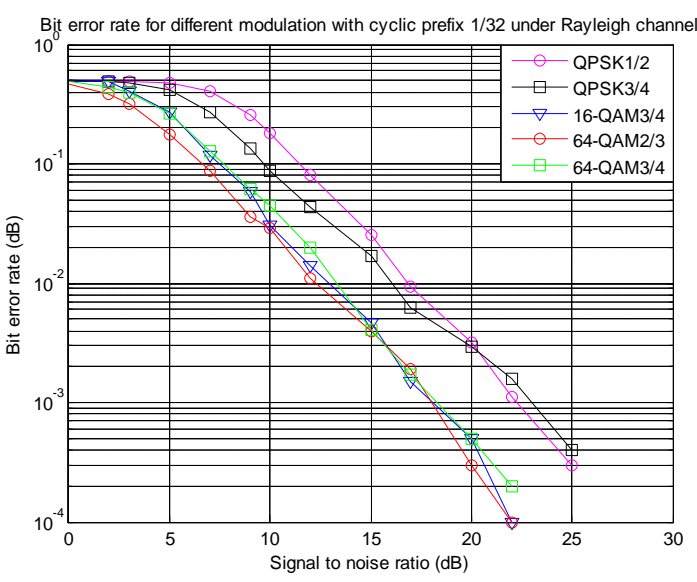

Fig. 10 BER of WiMAX with cyclic prefix 1/32

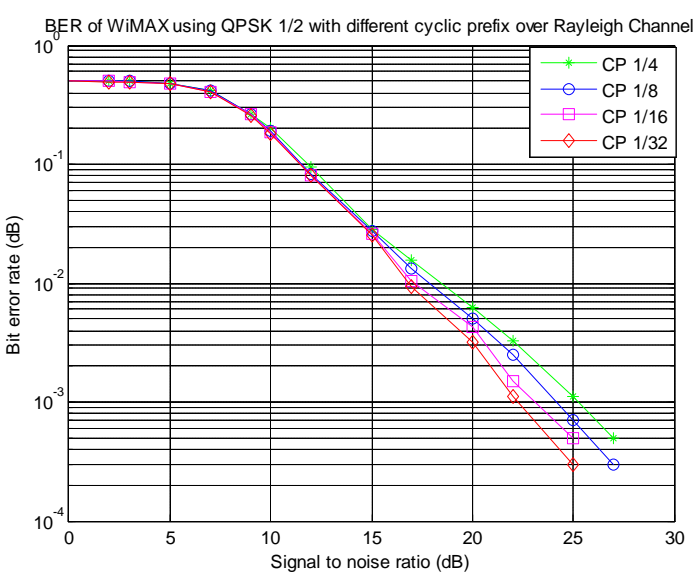

Fig. 11 BER of WiMAX using QPSK $1 \frac{1}{2}$

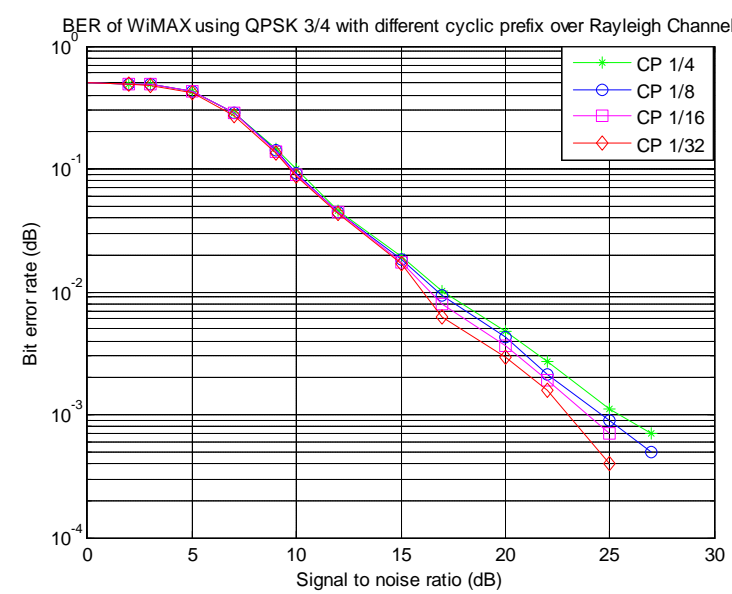

Fig. 12 BER of WiMAX using QPSK $3 / 4$ 


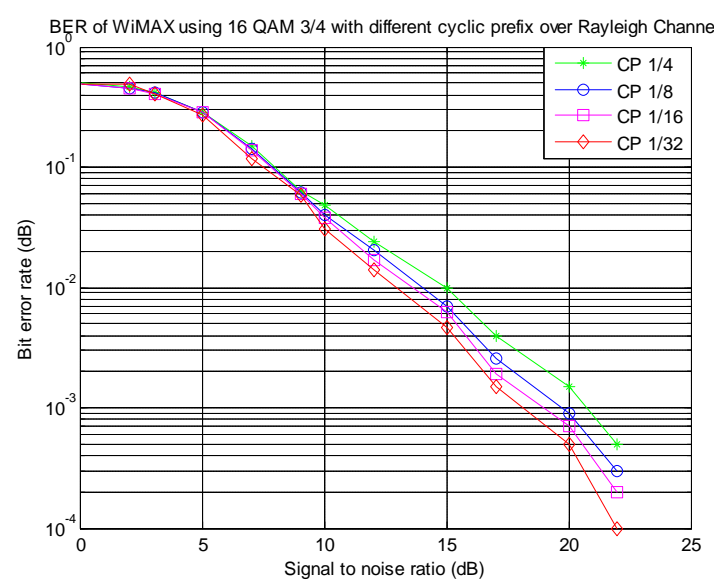

Fig. 13 BER of WiMAX using 16 QAM 3/4

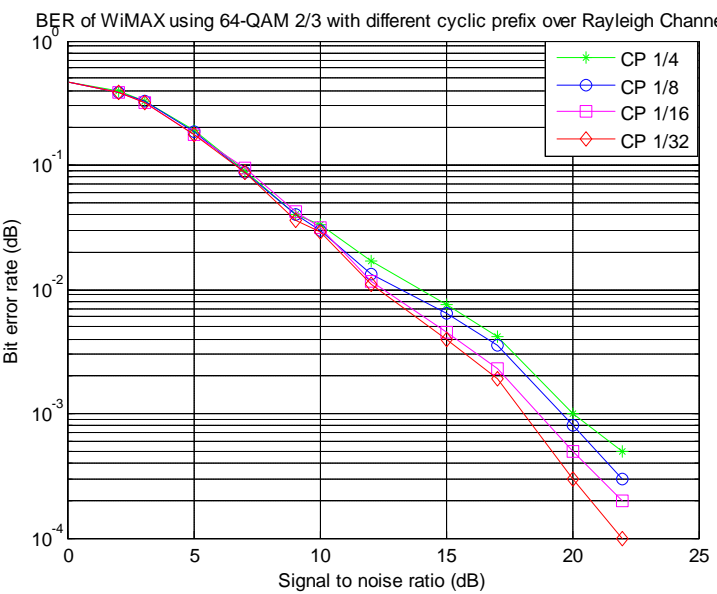

Fig. 14 BER of WiMAX using 64 QAM 2/3

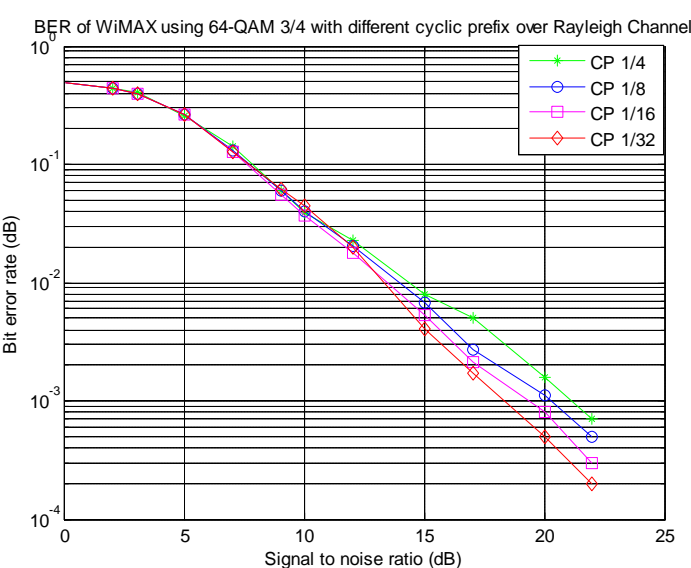

Fig. 15 BER of WiMAX using 64 QAM $3 / 4$

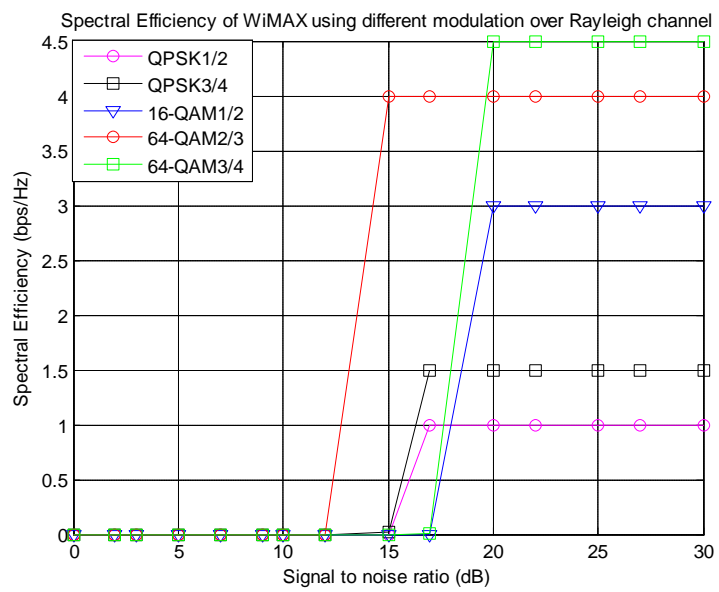

Fig. 16 Spectral Efficiency of WiMAX

Table 2 SNR requirement in WiMAX

\begin{tabular}{|c|c|c|c|c|}
\hline \multirow{2}{*}{ Modulation } & \multicolumn{4}{|c|}{ SNR(dB) required to achieve a BER of 10 } \\
\cline { 2 - 5 } & \multicolumn{4}{|c|}{ Cyclic Prefix } \\
\cline { 2 - 5 } & $\mathbf{1 / 4}$ & $\mathbf{1 / 8}$ & $\mathbf{1 / 1 6}$ & $\mathbf{1 / 3 2}$ \\
\hline QPSK 1/2 & 25.24 & 24.15 & 23.10 & 22.22 \\
\hline QPSK 3/4 & 25.42 & 24.62 & 23.92 & 23.01 \\
\hline 16 QAM 3/4 & 20.73 & 19.70 & 18.92 & 18.10 \\
\hline 64 QAM 2/3 & 20 & 19.54 & 18.63 & 18.04 \\
\hline 64 QAM 3/4 & 21.13 & 20.24 & 19.30 & 18.30 \\
\hline
\end{tabular}

Table 3 Spectral Efficiency of WiMAX over Rayleigh channel 


\begin{tabular}{|c|c|c|c|}
\hline \multicolumn{5}{|c|}{ Spectral Efficiency of WiMAX over Rayleigh channel } \\
\hline Modulation \& Code rate & Bits/Symbol & Spectral Efficiency & SNR(dB) \\
\hline QPSK 1/2 & 2 & 1 & From 17 onwards \\
\hline QPSK 3/4 & 2 & 2 & From 17 onwards \\
\hline 16 QAM 3/4 & 4 & 3 & From 20 onwards \\
\hline 64 QAM 2/3 & 6 & 4 & From 15 onwards \\
\hline 64 QAM 3/4 & 6 & 4.5 & From 20 onwards \\
\hline
\end{tabular}

\section{CONCLUSION}

In this paper, we have analyzed the BER vs. SNR and Spectral efficiency vs. SNR of OFDM based WiMAX physical layer using diverse modulation techniques and guard time intervals over Rayleigh channel. In Rayleigh channel, there is no line of sight from transmitter to receiver and also there are numerous impediments in the way. We can conclude that the results are different by utilizing different modulation techniques in OFDM based WiMAX physical layer system. Among all the simulations, 64 QAM R2/3 has lowest BER and QPSK R3/4 has the highest BER than other modulation techniques. The results also shows that the different modulations and coding rate give better performance with less SNR at cyclic prefix 1/32. The simulation outcomes of spectral efficiency shows that QPSK R1/2 has the lowest spectral efficiency and 64 QAM R3/4 has the highest spectral efficiency so for transmitting maximum amount of data, 64 QAM R3/4 can be used as it has lesser transmission error and higher spectral efficiency.

\section{REFERENCES}

[1] Jeffrey G. Andrews, Arunabha Ghosh, Rias Muhamed, "Fundamentals of WiMAX: Understanding Broadband Wireless Networking”, Prentice Hall, 2007.

[2] Loutfi Nuaymi, "WiMAX: Technology for Broadband Wireless Access", John Wiley \& Sons, 2007

[3] Mondal, Md. Ashraful Islam and Riaz Uddin. "Performance Evaluation of WiMAX Physical Layer under Addaptive Modulation Techniques and commmunication channels." International Journal of computer Science and Information Security, 2009: Vol. 5, No.1.

[4] Md. Anamul Islam, Md. Mobarak Hossain. "Spectral Efficiency and BER Performance Analysis of an IEEE 802.16 Standard WiMAX Physical Layer using Adaptive Modulation and Coding under AWGN and Fading Channels" Journal of Computing,
2010.

[5] M.A.Mohamed, F.W. Zaki and R.H. Mosbeh. "Simulation of WiMAX Physical LAyer:IEEE 802.16e." International Journal of Computer Science and Network Security, 2010: Vol. 10 No.11.

[6] Prabhakar Telagarapu, G.B.S.R. Naidu and K. Chiranjeevi. "Analysis of Coding Techniques in WiMAX." International Journal of Computer Applications, 2011: Vol. 22 No.3.

[7] Islam, Md. Ashraful Islam and A.Z.M. Touhidul. "Performance of WiMAX Physical Layer with Variations in Channel Coding and Digital Modulation under realistic Channel Conditions." International Journal of Information Sciences and Techniques, 2012: Vol.2,No.4.

[8] Abdul Rehman, Tauheed Khan amd Sunil Kumar Chaudhry. "Study of WiMAX Physical Layer under Adaptive Modulation Technique using Simulink." International Journal of Scientific Research Engineering and Technology, 2012: Vol 1 Issue 5 pp 005-011.

[9] Nuzhat Tasneem Awon, Md.Mizanur Rahman,Md. Ashraful Islam, A.Z.M Touhidul Islam. "Effects of AWGN and fading(rayleigh and Rician) channels on BER performance of a WiMAX communication system." International journal of computer science and information security 10, no. No.8 (August 2012).

[10] AlSlmman, Karrar, Haider M.AlSabbagh, and Saod A. Alseyab. "Performance Analysis for WiMAX Networks with employing LDPC code and RS-CC." Journal of Mobile communication, 2012: 28-37.

[11] Kushwah, Valmik Tilwari and Aparna Singh. "Performance Analysis of WiMAX 802.16e Physical Layer using Digital Modulation Techniques and Code Rates." International Journal of Engineering 
Research and Aplications, 2013: 1449-1454.

[12] Manju Agrawal, Naresh Purohit, Surabhi Kaushik. "Performance enhancement of IEEE 802.16e(Mobile WiMAX) system with adaptive modulation and coding technique. ." International Journal of Engineering Science and Research Technology, 2015.

International Journal of Engineering Research and Applications (IJERA) is UGC approved Journal with S1. No. 4525, Journal no. 47088. Indexed in Cross Ref, Index Copernicus (ICV 80.82), NASA, Ads, Researcher Id Thomson Reuters, DOAJ.

Bindu Handa. "Spectral Efficiency and Bit Error Rate Analysis of WiMAX Using Diverse Modulation Techniques over Rayleigh Channel." International Journal of Engineering Research and Applications (IJERA) 7.7 (2017): 06-13. 